Relations industrielles

Industrial Relations

\title{
Manpower Economics, by Edward B. Jakubauskas and Neil A. Palomba, Don Mills, Ontario, Addison-Wesley Publishing Company, 1973, 296 pp.
}

\section{Jean Sexton}

\section{Volume 29, numéro 4, 1974}

URI : https://id.erudit.org/iderudit/028577ar

DOI : https://doi.org/10.7202/028577ar

Aller au sommaire du numéro

Éditeur(s)

Département des relations industrielles de l'Université Laval

ISSN

0034-379X (imprimé)

1703-8138 (numérique)

Découvrir la revue

Citer ce compte rendu

Sexton, J. (1974). Compte rendu de [Manpower Economics, by Edward B. Jakubauskas and Neil A. Palomba, Don Mills, Ontario, Addison-Wesley Publishing Company, 1973, 296 pp.] Relations industrielles / Industrial Relations, 29(4), 897-897. https://doi.org/10.7202/028577ar

Tous droits réservés @ C Département des relations industrielles de l'Universite Laval, 1974
Ce document est protégé par la loi sur le droit d'auteur. L'utilisation des services d’Érudit (y compris la reproduction) est assujettie à sa politique d'utilisation que vous pouvez consulter en ligne.

https://apropos.erudit.org/fr/usagers/politique-dutilisation/ 
Manpower Economics, by Edward B. Jakubauskas and Neil A. Palomba, Don Mills, Ontario, Addison-Wesley Publishing Company, 1973, 296 pp.

Voici un nouvel ouvrage qui devrait retenir l'attention de ceux qui s'intéressent à l'enseignement de l'économique du travail. En effet, les auteurs n'utilisent pas l'expression «manpower economics » seulement parce que c'est maintenant la mode aux Etats-Unis. Ils vont fondamentalement plus loin que l'approche traditionnelle en économique du travail pour présenter systématiquement le processus d'utilisation et de développement de la main-d'œuvre. $\mathrm{Ce}$ livre est donc plus que de l'économique du travail, c'est de l'économique de la main-d'œuvre.

Cet ouvrage de base est très bien structuré et représente un instrument d'enseignement intéressant. Pour être logique avec leur approche d'économique de la main-d'œuvre, les auteurs présentent d'abord en première partie l'économique de la main-d'œuvre comme partie de l'économique du travail. C'est ici que les auteurs s'attardent sur les concepts de capital humain, de taux de rendement, etc.

La deuxième partie de Manpower Economics couvre ce dont tout bon livre d'économique du travail traite normalement : la détermination du niveau général de l'emploi, l'offre et la demande de travail, la détermination des salaires, la définition et la structure des marchés du travail, ainsi que la mobilité du travail. Cette deuxième partie est très bien faite.

C'est à partir de la troisième section que cet ouvrage sort réellement des sentiers normalement couverts par l'économique du travail. C'est une partie très importante dans la structure générale de l'ouvrage. En effet, après avoir présenté la théorie économique de la maind'œuvre surtout dans la partie II, les auteurs s'attaquent aux concepts de chômage et de vacances d'emploi, aux tendances de chômage, au sous-emploi et à la pauvreté avant d'aborder le problème des politiques publiques dans le domaine de la main-d'œuvre. On doit noter au moins deux sujets très bien traités dans cette section : le concept de chômage et la mesure de tendances de chômage par des indices de dispersion.
C'est dans la quatrième partie de Manpower Economics que les auteurs traitent de la politique publique dans le domaine de la main-d'œuvre aux EtatsUnis. Le concept de politique de maind'œuvre, les développements de la politique de main-d'œuvre américaine, l'évaluation et la planification des programmes et des propositions pour combattre la pauvreté sont autant de sujets que les auteurs abordent dans cette section. Si nous avions quelque chose à reprocher aux auteurs, ce serait sur deux points précis de cette section. D'abord, leur discussion de la nature de la politique de main-d'œuvre peut laisser à désirer. En effet, cette discussion est beaucoup trop brève et reste incomplète surtout par le manque de référence systématique à la littérature publiée depuis Harbison (1964) sur lequel les auteurs s'appuient ouvertement. En second lieu, le chapitre 15 sur l'évaluation des programmes de main-d'œuvre prête énormément à la critique. D'abord les auteurs n'établissent pas le lien entre l'évaluation des programmes de main-d'œuvre et les principes généraux de la recherche évaluative (Suchman, Weiss, Borus, Hardin, etc.). Ce reproche est d'autant plus sérieux que cet ouvrage se veut un livre pour l'introduction à l'économique de la main-d'œuvre. Ensuite, même si l'attention s'est surtout centrée à date aux USA sur l'évaluation de la formation professionlelle, ce n'est pas une raison pour présenter l'évaluation comme si c'était seulement ce programme qui devait être évalué. Les différences entre les programmes exigent des adaptations dans les méthodologies. Ce n'est pas ici qu'on les trouvera. Finalement, les auteurs ne présentent pas assez complètement les différentes techniques d'évaluation. L'analyse coûts-bénéfices n'est pas la seule technique.

En guise de conclusion à cet ouvrage, les auteurs, dans un effort de synthèse, prônent l'intégration des politiques d'emploi et de main-d'œuvre pour réduire la prétendue relation d'arbitrage entre le chômage et l'inflation.

En résumé, Manpower Economics est un livre généralement bien fait et très bien structuré pour un cours d'introduction aux problèmes économiques de la main-d'œuvre.

Université Laval
Jean SEXTON 